\title{
Strontium Chloride: Can It Be a New Treatment Option for Ulcerative Colitis?
}

\author{
Firdevs Topal, ${ }^{1}$ Ozlem Yonem, ${ }^{2,3}$ Nevin Tuzcu, ${ }^{4}$ Mehmet Tuzcu, ${ }^{5}$ \\ Hilmi Ataseven, ${ }^{6}$ and Melih Akyol ${ }^{7}$ \\ ${ }^{1}$ Gastroenterology Clinic, Ataturk Training and Research Hospital, 35965 Izmir, Turkey \\ ${ }^{2}$ Department of Gastroenterology, Faculty of Medicine, Cumhuriyet University, 58140 Sivas, Turkey \\ ${ }^{3}$ Cumhuriyet Universitesi Hastanesi, Gastroenteroloji B.D., 58140 Sivas, Turkey \\ ${ }^{4}$ Department of Pharmaceutical Microbiology, Faculty of Pharmacy, Cumhuriyet University, 58140 Sivas, Turkey \\ ${ }^{5}$ Department of Pathology, Faculty of Veterinary Medicine, Cumhuriyet University, 58140 Sivas, Turkey \\ ${ }^{6}$ Gastroenterology Clinic, Anadolu Hospital, 58070 Sivas, Turkey \\ ${ }^{7}$ Department of Dermatology, Faculty of Medicine, Cumhuriyet University, 58140 Sivas, Turkey
}

Correspondence should be addressed to Ozlem Yonem; oyonem@cumhuriyet.edu.tr

Received 26 February 2014; Revised 11 May 2014; Accepted 20 May 2014; Published 18 June 2014

Academic Editor: Guang-Yin Xu

Copyright (C) 2014 Firdevs Topal et al. This is an open access article distributed under the Creative Commons Attribution License, which permits unrestricted use, distribution, and reproduction in any medium, provided the original work is properly cited.

\begin{abstract}
Background/Aims. Patients with ulcerative colitis still need effective therapy without major side effects. It has been found that strontium can suppress NF $\kappa$ B activation induced by TNF- $\alpha$. This opens a gate to a new anti-TNF agent which is cheap and can be given orally. We for the first time aimed to investigate the effect of strontium chloride $\left(\mathrm{SrCl}_{2}\right)$ on inflammation in experimental colitis. Methods. Thirty female Wistar albino rats were divided into 5 groups each containing 6 rats. The rats in groups 1 and 2 served as the healthy control and colitis group, respectively. The rats in groups 3, 4, and 5 had colitis and received $40 \mathrm{mg} / \mathrm{kg} \mathrm{SrCl}{ }_{2}$, $160 \mathrm{mg} / \mathrm{kg} \mathrm{SrCl}_{2}$, and $1 \mathrm{mg} / \mathrm{kg}$ prednisolone by oral gavage, respectively. The rats were sacrificed for histological evaluation and determination of serum neopterin, TNF- $\alpha$, and IFN- $\gamma$ levels. Results. The neopterin, TNF- $\alpha$ and IFN $\gamma$ levels of group 2 was significantly higher than the other groups. The neopterin, TNF- $\alpha$, and IFN- $\gamma$ levels of controls and other treatment groups were comparable. There were a significant difference in macroscopic and microscopic healing between group 2 and other groups histologically. But there was not a significant difference within treatment receiving groups. Conclusion. $\mathrm{SrCl}_{2} \mathrm{had}_{\mathrm{C}} \mathrm{comparable}$ therapeutic efficiency with prednisolone.
\end{abstract}

\section{Introduction}

Inflammatory bowel disease is a chronic intestinal inflammatory condition, the pathogenesis of which is complex and not fully understood. It includes mainly two diseases, namely, ulcerative colitis and Crohn's disease, that still need effective and lasting therapy without major side effects.

Available evidence suggests that an abnormal immune response against the microorganisms of the intestinal flora is responsible for the disease in genetically susceptible individuals [1]. Increases in the production of proinflammatory cytokines by both lymphocytes and macrophages have also been implicated in the pathogenesis of inflammatory bowel diseases [2]. NF- $\kappa \mathrm{B}$ activation is seen in mucosal biopsy specimens from patients with active Crohn's disease and ulcerative colitis. Treatment of patients with inflammatory bowel diseases with steroids decreases NF- $\kappa \mathrm{B}$ activity in biopsy specimens and reduces clinical symptoms. These results suggest that stimulation of the NF- $\kappa \mathrm{B}$ pathway may be involved in the enhanced inflammatory response associated with these diseases [3].

Strontium salts are used in routine clinical practice for treatment of osteoporosis, sensitive teeth, and bone pain related to bone metastasis [4-6]. Recently strontium was found to block TNF- $\alpha$ induced NF $\kappa$ B signal transduction and in another article it was found to inhibit inflammatory 
mediators production by human monocytes [7-9]. All of these developments make strontium suitable for use in inflammatory diseases. For this reason for the first time in the literature we investigated the effect of $\mathrm{SrCl}_{2}$ on inflammation in experimental colitis and compared it with a novel therapeutic agent prednisolone.

\section{Methods}

2.1. Ethics. The present study was approved by the Animal Ethics Committee of Cumhuriyet University in accordance with the Standards for the Care and Use of Laboratory Animals.

2.2. Animals. Thirty female Wistar albino rats each weighing about 180-200 grams were included in the study. They were maintained under standard conditions (12 h light/dark cycle; $25 \pm 3^{\circ} \mathrm{C}, 45-65 \%$ humidity) and had free access to standard rat feed and water ad libitum. The experiments were performed during the light portion between 08:00 and 12:00 a.m. to avoid circadian influences.

2.3. Serum Proinflammatory Cytokines. Serum levels of neopterin (Rat Neopterin ELISA- Cusabio,), TNF- $\alpha$ (Rat TNF- $\alpha$ Platinum ELISA- eBioscience), and IFN- $\gamma$ (Rat IFN- $\gamma$ Platinum ELISA- eBioscience) were determined in the groups by ELISA method. Thermo Multiskan device was used for ELISA applications.

2.4. Induction of Colitis. Experimental ulceration in colon tissue was done according to the method described by Mousavizadeh et al [10] with slight modification. Under light ether anesthesia rats were administered $2 \mathrm{~mL}$ of $4 \%$ acetic acid solution in ethanol by transrectally using a $(2.7 \mathrm{~mm})$ soft pediatric catheter. After acetic acid administration, rats were holed horizontally for 2 minutes to prevent acetic acid leakage. Then the animals were divided into 5 groups each containing 6 rats. The rats in group 1 served as the healthy control group and we did not induce colitis in them. We induced colitis in groups $2-5$. The rats in group 2 served as the colitis group which received no medication; the rats in group 3 had colitis and received $40 \mathrm{mg} / \mathrm{kg}$ strontium chloride; the rats in group 4 had also colitis but received $160 \mathrm{mg} / \mathrm{kg}$ strontium chloride; the rats having colitis in group 5 received $1 \mathrm{mg} / \mathrm{kg}$ prednisolone. All these treatments were given for seven days orally by using oral gavage.

Seven days after the induction of colitis, rats in all groups including groups 1-5 were sacrificed by cervical subluxation, blood was obtained by retroorbital puncture for biochemical estimation, and the colon was removed and postfixed. Sections of the colon were stained for hematoxylin-eosin and evaluated microscopically and scored for inflammation.

\subsection{Histology}

2.5.1. Macroscopy. After sacrificing animals, the colon was carefully dissected from anus till rectum and placed in saline. The colon was opened longitudinally over the mesenteric border and washed gently in saline. It was placed on a rubber mat with the mucosal side up and evaluated macroscopically. Macroscopic changes in colonic mucosa were scored from 0 to 3 with score 0 referred to preserved mucosa with normal appearance, score 1 to edema and congestion, score 2 to ulceration and hemorrhage, and finally score 3 to abundant multiple ulcers and thinned bowel wall.

2.5.2. Microscopy. After macroscopic evaluation, the colon was then placed with the serosal side up and dissected. Serial sections of $6 \mathrm{~mm}$ were obtained and fixed with \%10 buffered formaldehyde. They were embedded in paraffin blocks. Sections of $5 \mu \mathrm{m}$ were prepared from these blocks and stained with hematoxylin and eosin and scored for inflammation. Severity and abundance of edema, necrosis, inflammatory infiltration, apoptosis, thrombosis-vascular congestion, and shedding of epithelial cells were considered during microscopic scoring. Histopathological evaluation of the slides was performed by one pathologist who was blinded about the groups. Microscopic changes were scored from 0 to 3 with score 0 referred to no swelling in epithelial cells, normal crypt appearance, sporadic monocyte infiltration, either sporadic or none neutrophilic infiltration; score 1 to moderate blurred swelling in epithelial cells, sporadic polymorphonuclear leukocyte infiltration in crypts, mild monocyte, and neutrophil infiltration in submucosa; score 2 to shedding of epithelial cells and flatness of mucosa, shedding of crypt epithelium, moderate monocytic-neutrophilic infiltration of submucosa; and score 3 to marked epithelial ulcers, crypt abscesses, and marked monocytic and neutrophilic infiltration of submucosa.

2.6. Statistical Analysis. Data were analyzed by using SPSS 15.0 package software. As the assumptions of parametric tests could not be fulfilled, Kruskal Wallis, Mann-Whitney U test, and Chi-square tests were used for statistical evaluation. A $P$ value of $<0.05$ was considered to be significant.

\section{Results}

3.1. Serum Proinflammatory Cytokines. We compared the mean serum neopterin, TNF- $\alpha$, and interferon- $\gamma$ levels of groups after 7 days of treatment.

The neopterin, TNF- $\alpha$, and interferon- $\gamma$ levels of colitis group (group 2) were significantly higher than those of the group 1 (healthy control), group 3 (40 mg/kg: low dose strontium), group 4 (160 mg/kg: high dose strontium), and group 5 (prednisolone) $(P<0.05)$. The neopterin, TNF- $\alpha$, and interferon- $\gamma$ levels of controls, low dose strontium, high dose strontium, and prednisolone groups were comparable $(P>0.05)$ (Figures 1,2 , and 3$)$.

3.2. Histopathological Studies. There was a significant difference by means of both macroscopic and microscopic scores between group 2 (The colitis group) and other four groups $(P<0.05)$. But there was no significant difference within treatment receiving groups (groups 3, 4 and 5) when compared in pairs $(P>0.05)$. Table 1 shows the macroscopic 


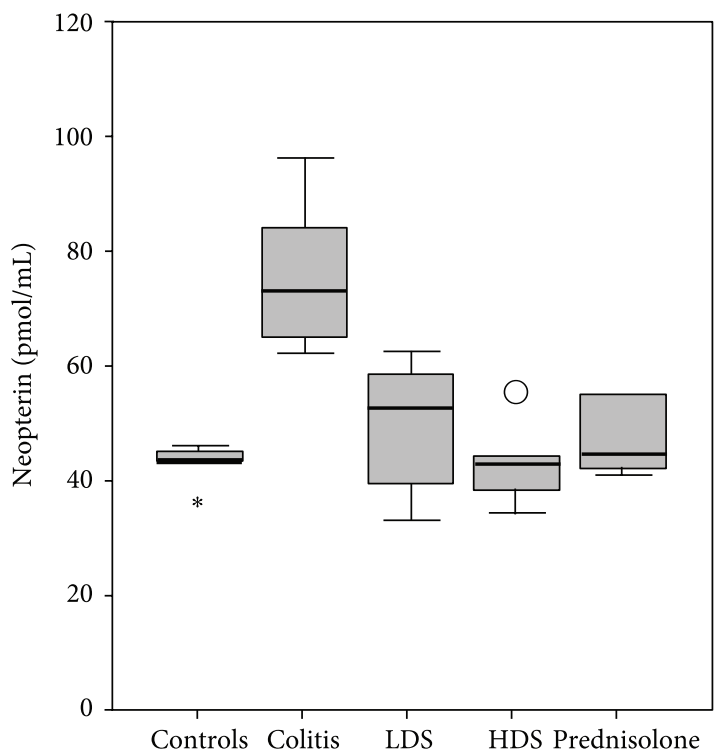

Figure 1: Comparison of neopterin levels among groups. The horizontal lines in the middle of each box indicate the median, while the top and bottom borders of the box mark the 25th and 75th percentiles, respectively. The whiskers above and below the box mark the maximum and minimum neopterin levels. Open circles indicate outliers. Asterisks represent extreme cases.

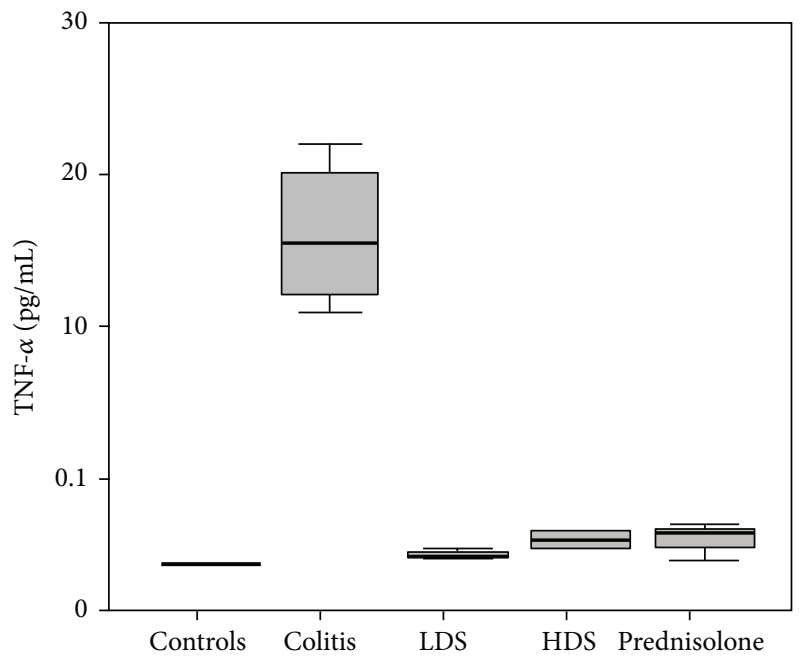

FIgURE 2: Comparison of TNF-alpha levels among groups. The horizontal lines in the middle of each box indicate the median, while the top and bottom borders of the box mark the 25th and 75 th percentiles, respectively. The whiskers above and below the box mark the maximum and minimum TNF-alpha levels.

and microscopic scores of all rats involved in this study. Figure 4 shows the histopathological changes in the colon of experimental rats.

\section{Discussion}

We have found in our study for the first time in the literature that strontium chloride had comparable therapeutic efficiency when compared with prednisolone in a rat ulcerative

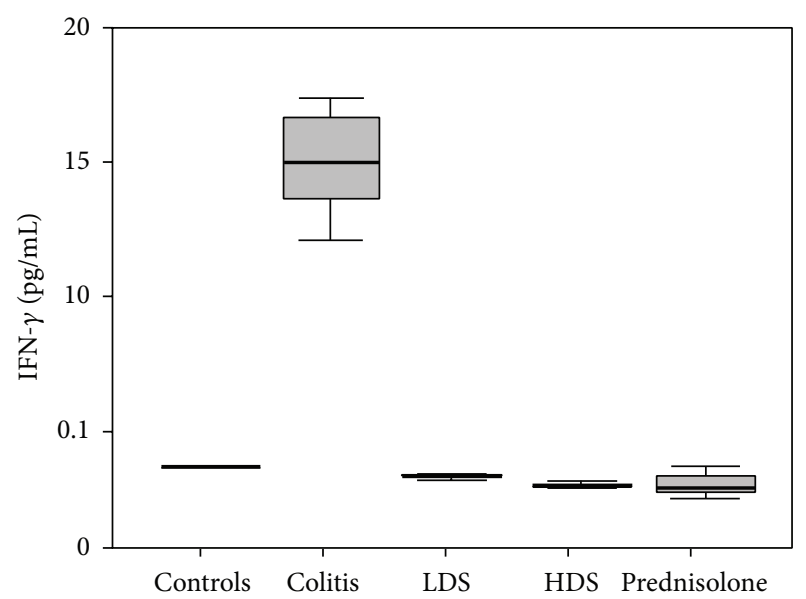

FIgURE 3: Comparison of IFN-gamma levels among groups. The horizontal lines in the middle of each box indicate the median, while the top and bottom borders of the box mark the 25th and 75th percentiles, respectively. The whiskers above and below the box mark the maximum and minimum IFN-gamma levels.

colitis model. We investigated the effect of strontium at two doses, $40 \mathrm{mg} / \mathrm{kg}$ and $160 \mathrm{mg} / \mathrm{kg}$, but did not find a difference between these two doses by means of histological and biochemical healing.

The immune system plays a dominant role in inflammatory bowel disease. During active inflammation, Th0 cells differentiate into T helper cell type (Th)1, Th17, and/or Th2 cells, upon the action of interleukin- (IL-) 12/IL-18, IL$6 /$ TGF- $\beta$, or IL- 4 , respectively. Th1 cells, in turn, produce vast amounts of interferon- (IFN-) $\gamma$ and tumor necrosis factor(TNF-) $\alpha$. IFN- $\gamma$ induces the production of further TNF- $\alpha$ by intestinal activated macrophages and triggers apoptosis of epithelial cells. TNF- $\alpha$ promotes the differentiation of lamina propria stromal cells into activated myofibroblasts, which start producing high quantities of tissue-degrading matrix metalloproteinases (MMPs). MMPs degrade the extracellular matrix and the basement membrane, thus inducing enterocyte apoptosis [1].

Neopterin, a pyrazino[2,3-d]-pyrimidine compound, is a metabolite of cyclic guanosine monophosphate that is released by activated dendritic cells and macrophages after induction by $\gamma$ interferon [11]. Neopterin concentration increase in the blood reflects the activation of cellular immunity and it has been suggested that it is an excellent marker for the monocyte/macrophage axis in some clinical situations [12]. We have found the serum TNF- $\alpha$, IFN- $\gamma$, and neopterin values to be significantly increased in our rat colitis model.

Strontium salts may also directly act on nonneuronal cells such as keratinocytes or immunoregulatory inflammatory cells. Recent reports demonstrate that strontium salts can suppress keratinocyte derived tumor necrosis factor-alpha (TNF- $\alpha$ ), interleukin- $1 \alpha$ (IL- $1 \alpha$ ), and IL-6 in in vitro cultures [13]. It has also been shown that strontium can suppress $\mathrm{NF} \kappa \mathrm{B}$ activation induced by TNF- $\alpha$ and can inhibit inflammatory mediators production by human monocytes [7-9]. Considering all these effects of strontium, we hypothesized that it can 


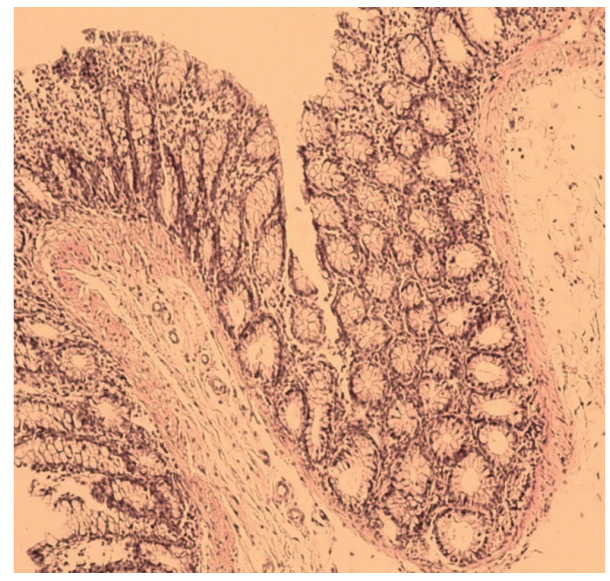

(a)

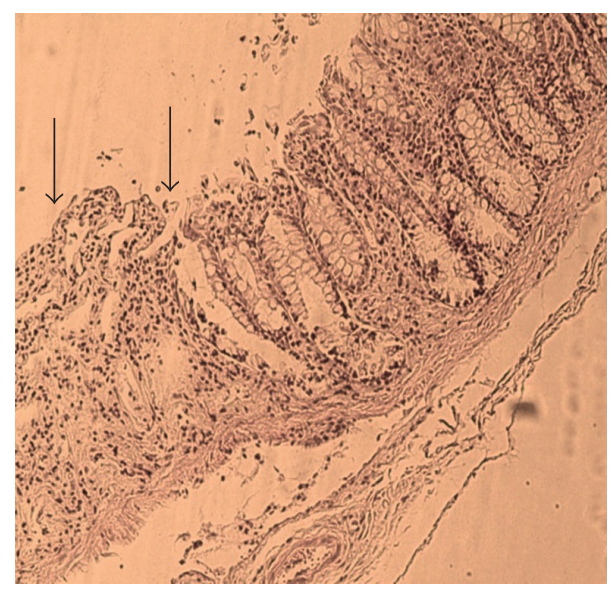

(c)

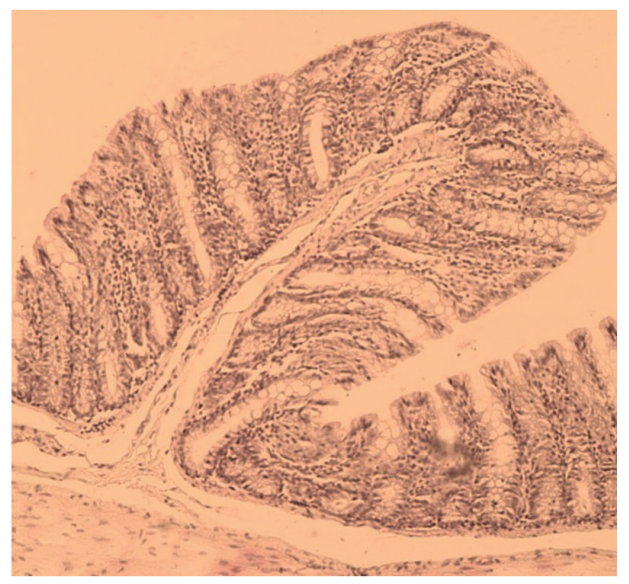

(b)

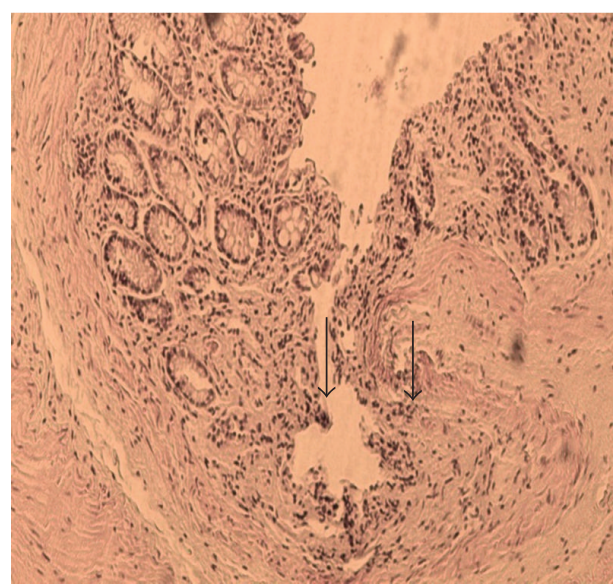

(d)

FIGURE 4: Histopathological changes in the colon of experimental rats. (a) Normal intact mucosa from normal colon, hematoxylin/eosin $\times 260$. (b) Appearance of colon in low-dose strontium group, hematoxylin/eosin $\times 400$. (c) Arrows show healed ulcer in the low dose strontium group, hematoxylin/eosin $\times 260$. (d) Appearance of colon in the ulcerative colitis group; arrows show ulcers, hematoxylin/eosin $\times 260$.

TABLE 1: Macroscopic and microscopic scores of the each 6 rats in the five groups.

\begin{tabular}{|c|c|c|c|c|c|}
\hline & Group 1 & Group 2 & Group 3 & Group 4 & Group 5 \\
\hline \multirow{6}{*}{$\begin{array}{l}\text { Macroscopic } \\
\text { scores }\end{array}$} & 0 & 3 & 2 & 1 & 1 \\
\hline & 0 & 3 & 1 & 1 & 2 \\
\hline & 0 & 3 & 2 & 2 & 1 \\
\hline & 0 & 3 & 0 & 2 & 2 \\
\hline & 0 & 3 & 2 & 2 & 2 \\
\hline & 0 & 3 & 0 & 1 & 2 \\
\hline Median \pm SEM & 0 & 3 & $1.50 \pm 0.40$ & $1.50 \pm 0.22$ & $2.00 \pm 0.21$ \\
\hline \multirow{6}{*}{$\begin{array}{l}\text { Microscopic } \\
\text { scores }\end{array}$} & 0 & 3 & 1 & 2 & 1 \\
\hline & 0 & 3 & 1 & 1 & 2 \\
\hline & 0 & 3 & 2 & 2 & 1 \\
\hline & 0 & 2 & 1 & 1 & 1 \\
\hline & 0 & 3 & 1 & 1 & 0 \\
\hline & 0 & 3 & 0 & 2 & 2 \\
\hline Median \pm SEM & 0 & 3 & $1.00 \pm 0.25$ & $1.50 \pm 0.22$ & $1.00 \pm 0.30$ \\
\hline
\end{tabular}

${ }^{*}$ There was a significant difference by means of both macroscopic and microscopic scores between group 2 (The colitis group) and other four groups $(P<0.05)$. But there was no significant difference within treatment receiving groups (groups 3,4 , and 5 ) when compared in pairs $(P>0.05)$. 
be beneficial in inflammatory bowel disease. Macroscopically in histology all of our ulcerative colitis group rats received the score 3 which meant abundant multiple ulcers and thinned bowel wall; microscopically again all of our ulcerative colitis rats received the score 3 which meant marked epithelial ulcers, crypt abscesses, and marked monocytic and neutrophilic infiltration of submucosa. We observed the healing effect of strontium both macroscopically and microscopically that is comparable with prednisolone.

Strontium has been used to treat patients with osteoporosis or other disorders of bone mineralization. Treatment has involved administration of "low doses" of strontium salts (usually the lactate, gluconate, carbonate, or ranelate) over several years. No adverse side-effects were reported in studies on osteoporosis patients [14]. Beneficial effects on bone mineralization (a 17\% increase in mineral bone volume and a $70 \%$ increase in the number of bone-forming sites) and no adverse effect on the hydroxyapatite mineral particle size were observed in rats at a dose of $168 \mathrm{mg} / \mathrm{kg}$ [15]. And Kroes et al. investigated short-term toxicity of strontium chloride in rats at doses of 2.6, 10, 40, and $180 \mathrm{mg} / \mathrm{kg}$ [16]. We used strontium at two doses, namely, $40 \mathrm{mg} / \mathrm{kg}$ and $160 \mathrm{mg} / \mathrm{kg}$, and found no significant difference about the therapeutic efficiency of these two doses. New rat studies investigating the minimum effective strontium dose on colitis in rats can be planned.

In conclusion, we have found in our study that strontium chloride has comparable therapeutic efficiency with prednisolone in a rat ulcerative colitis model. We have found the dose of $40 \mathrm{mg} / \mathrm{kg}$ to be effective in suppressing inflammation in rats; however, new studies investigating the efficiency of lower doses can be planned. Our findings have important implications in that a new way to an alternative therapy for nonresponder patients is opened and a hope for osteoporotic ulcerative colitis patients emerged with one medication for two diseases. However, further studies in different phases are needed to confirm our results.

\section{Conflict of Interests}

The authors declare that there is no conflict of interests regarding the publication of this paper.

\section{References}

[1] A. Geremia, P. Biancheri, P. Allan, G. R. Corazza, and A. di Sabatino, "Innate and adaptive immunity in inflammatory bowel disease," Autoimmunity Reviews, vol. 13, pp. 3-10, 2014.

[2] D. K. Podolsky, "Medical progress: inflammatory bowel disease," The New England Journal of Medicine, vol. 325, no. 13, pp. 928-937, 1991.

[3] Y. Yamamoto and R. B. Gaynor, "Therapeutic potential of inhibition of the NF- $\kappa$ B pathway in the treatment of inflammation and cancer," The Journal of Clinical Investigation, vol. 107, no. 2, pp. 135-142, 2001.

[4] S. Tan, B. Zhang, X. Zhu et al., "Deregulation of bone forming cells in bone diseases and anabolic effects of strontiumcontaining agents and biomaterials," BioMed Research International, vol. 2014, Article ID 814057, 12 pages, 2014.
[5] B. Collaert and C. Fischer, "Dentine hypersensitivity: a review," Endodontics \& Dental Traumatology, vol. 7, no. 4, pp. 145-152, 1991.

[6] K. A. Autio, H. I. Scher, and M. J. Morris, "Therapeutic strategies for bone metastases and their clinical sequelae in prostate cancer," Current Treatment Options in Oncology, vol. 13, no. 2, pp. 174-188, 2012.

[7] C. Huang, L. Li, X. Yu et al., "The inhibitory effect of strontiumdoped calcium polyphosphate particles on cytokines from macrophages and osteoblasts leading to aseptic loosening in vitro," Biomedical Materials, vol. 9, no. 2, Article ID 025010, 2014.

[8] M. Yamaguchi and M. N. Weitzmann, "The intact strontium ranelate complex stimulates osteoblastogenesis and suppresses osteoclastogenesis by antagonizing NF- $\mathrm{B}$ activation," Molecular and Cellular Biochemistry, vol. 359, no. 1-2, pp. 399-407, 2012.

[9] E. Buache, F. Velard, E. Bauden et al., "Effect of strontiumsubstituted biphasic calcium phosphate on inflammatory mediators production by human monocytes," Acta Biomaterialia, vol. 8, no. 8, pp. 3113-3119, 2012.

[10] K. Mousavizadeh, R. Rahimian, G. Fakhfouri et al., "Antiinflammatory effects of 5-HT receptor antagonist, tropisetron on experimental colitis in rats," European Journal of Clinical Investigation, vol. 39, pp. 375-383, 2009.

[11] N. Husain, K. Tokoro, J. M. Popov, S. J. Naides, M. J. Kwasny, and A. L. Buchman, "Neopterin concentration as an index of disease activity in Crohn's disease and ulcerative colitis," Journal of Clinical Gastroenterology, vol. 47, no. 3, pp. 246-251, 2013.

[12] S. Pingle, R. Tumane, and A. Jawade, "Neopterin: biomarker of cell-mediated immunity and potent usage as biomarker in silicosis and other occupational diseases," Indian Journal of Occupational and Environmental Medicine, vol. 12, no. 3, pp. 107-111, 2008.

[13] P. Celerier, A. Richard, P. Litoux, and B. Dreno, "Modulatory effects of selenium and strontium salts on keratinocyte-derived inflammatory cytokines," Archives of Dermatological Research, vol. 287, no. 7, pp. 680-682, 1995.

[14] P. Watts and P. Howe, "Strontium and strontium compounds," Concise International Chemical Document 77, WHO Press, Geneva, Switzerland, 2010.

[15] M. D. Grynpas, E. Hamilton, R. Cheung et al., "Strontium increases vertebral bone volume in rats at a low dose that does not induce detectable mineralization defect," Bone, vol. 18, no. 3, pp. 253-259, 1996.

[16] R. Kroes, E. M. den Tonkelaar, A. Minderhoud et al., "Short term toxicity of strontium chloride in rats," Toxicology, vol. 7, no. 1, pp. 11-21, 1977. 


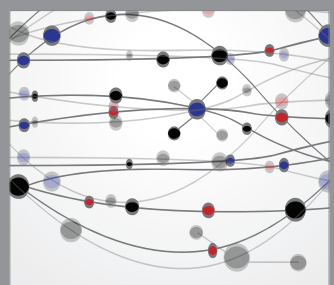

The Scientific World Journal
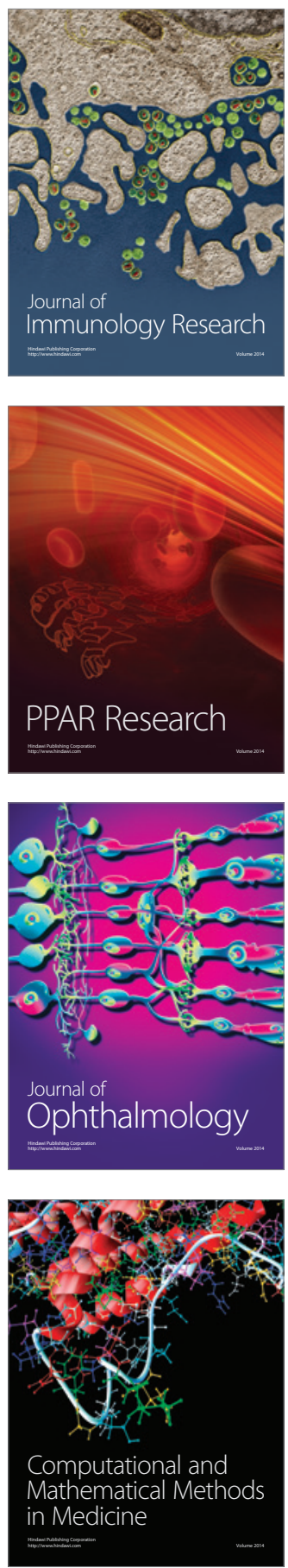

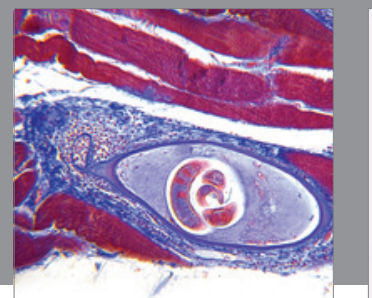

Gastroenterology

Research and Practice
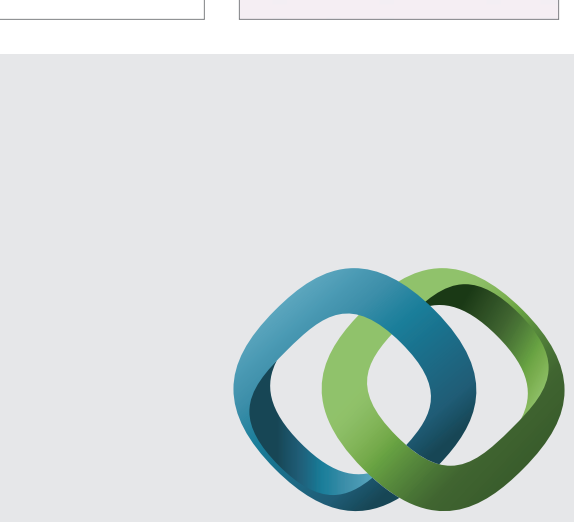

\section{Hindawi}

Submit your manuscripts at

http://www.hindawi.com
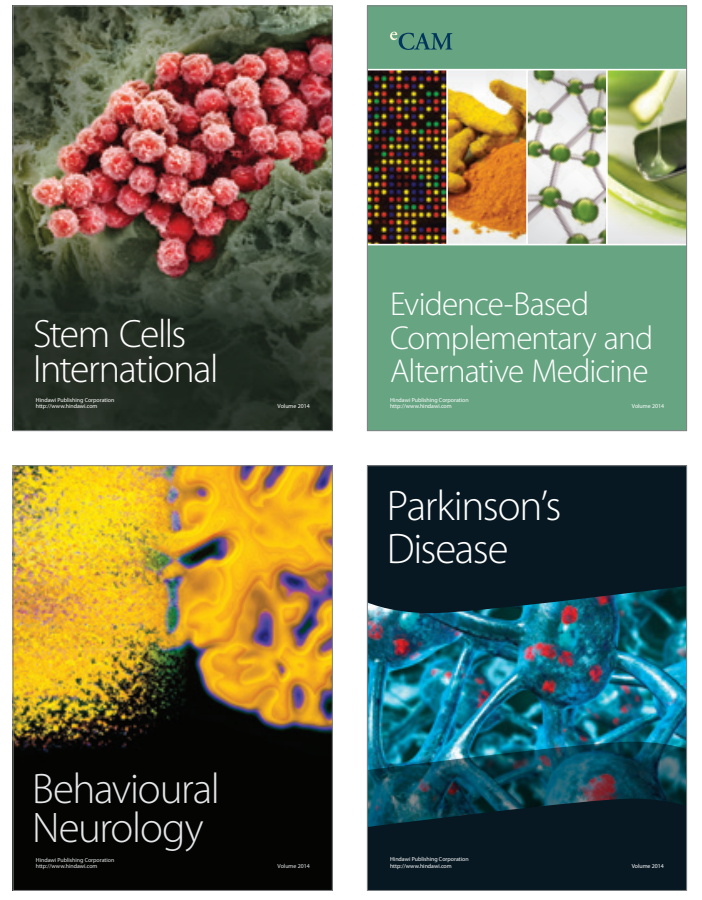
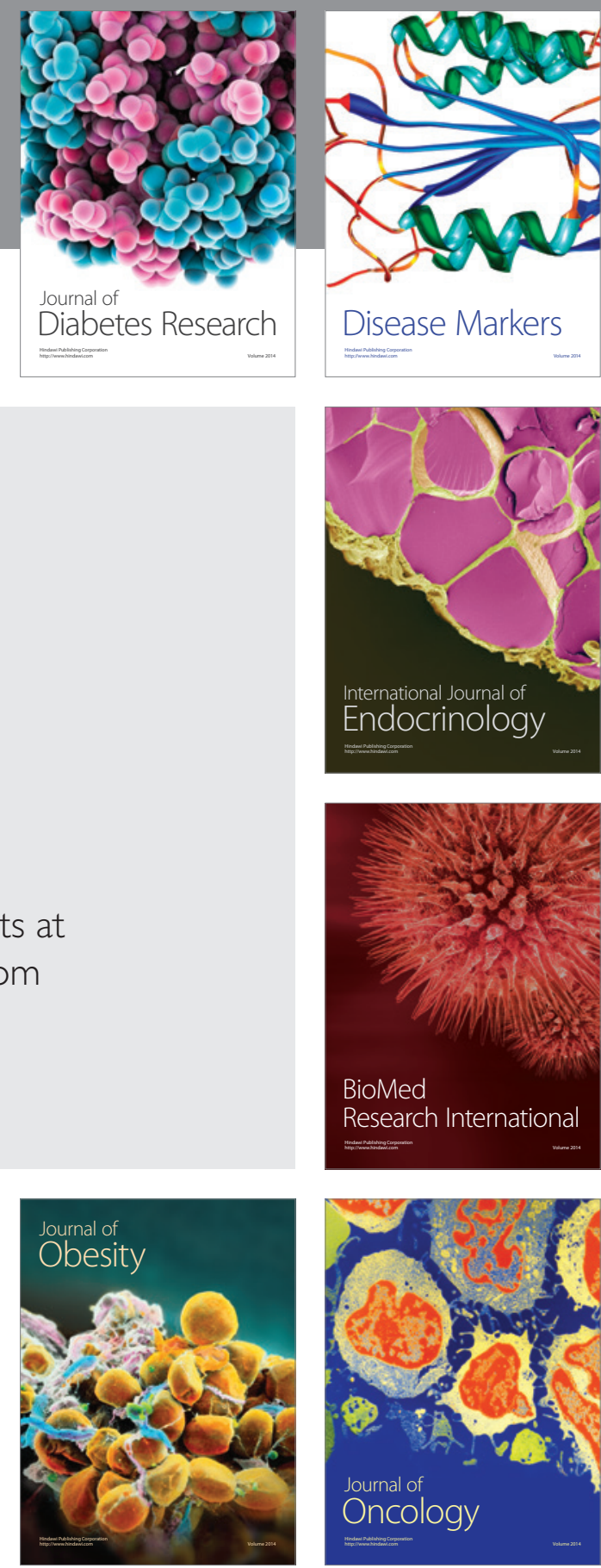

Disease Markers
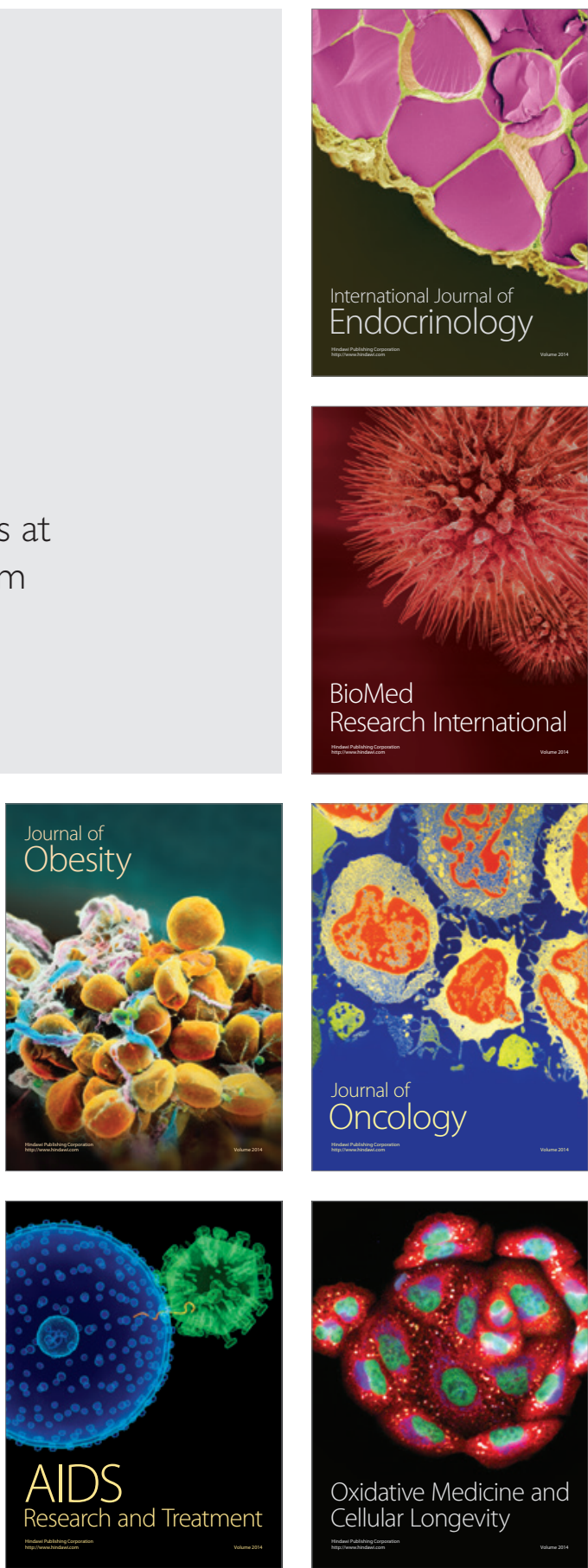